\title{
Association of atrophy of the medial temporal lobe with reduced blood flow in the posterior parietotemporal cortex in patients with a clinical and pathological diagnosis of Alzheimer's disease
}

\author{
K A Jobst, A D Smith, C S Barker, A Wear, E M King, A Smith, P A Anslow, \\ A J Molyneux, B J Shepstone, N Soper, K A Holmes, J R Robinson, R A Hope, \\ C Oppenheimer, K Brockbank, B McDonald
}

\begin{abstract}
A combination of medial temporal lobe atrophy, shown by computed tomography, and reduced blood flow in the parietotemporal cortex, shown by single photon emission tomography, was found in $86 \%(44 / 51)$ of patients with a clinical diagnosis of senile dementia of the Alzheimer type (SDAT). The same combination of changes was found in four out of 10 patients with other clinical types of dementia and in two out of 18 with no evidence of cognitive deficit. Of the 12 patients who died, 10 fulfilled histopathological criteria for Alzheimer's disease, nine of them having a clinical diagnosis of SDAT, and one a clinical diagnosis of multi-infarct dementia. All 10 patients with histopathologically diagnosed Alzheimer's disease had shown a combination of hippocampal atrophy and reduced parietotemporal blood flow in life. In 10 patients (nine with SDAT) out of 12 in whom the hippocampal atrophy was more noticeable on one side of the brain than on the other the parietotemporal perfusion deficit was also asymmetrical, being greater on the side showing more hippocampal atrophy. These results suggest that the combination of atrophy of the hippocampal formation and reduced blood flow in the parietotemporal region is a feature of dementia of the Alzheimer type and that the functional change in the parietotemporal region might be related to the loss of the projection neurons in the parahippocampal gyrus that innervate this region of the neocortex.
\end{abstract}

CT has shown that many patients with a clinical diagnosis of senile dementia of the Alzheimer type (SDAT) display more appreciable cerebral atrophy than expected for their age. ${ }^{1}$ Functional changes in the brains of demented patients have been found by methods that show cerebral blood flow, or uptake of glucose, oxygen or radioligands: the most frequent finding is a functional deficit in the posterior parietal and temporal lobes of the cortex. $^{2-5}$ Single photon emission tomography (SPET) has recently been used to show that the functional deficits in the posterior parietal and lateral temporal lobes are related to the degree of cognitive decline, ${ }^{6-8}$ but such findings are difficult to reconcile with neuropathological studies showing that the most severely affected parts of the brain in Alzheimer's disease are in the medial lobe, mainly the amygdala, the hippocampal formation and adjacent parahippocampal gyrus: ${ }^{9-13}$ Atrophy of these areas can be revealed in life by CT studies in which the scan angle is adjusted to give a clearer view of the medial temporal lobe, and such studies in patients with a clinical diagnosis of Alzheimer's disease have, indeed, shown severe damage to structures in the medial temporal lobe. ${ }^{14-16}$ However, atrophy of the medial temporal lobe is also found in other conditions-notably, epilepsy and hypoxia, ${ }^{17}$ schizophrenia, ${ }^{18}$ and amnesia ${ }^{19}$-and so is not unique to dementia of the Alzheimer's type.

In none of the CT and SPET studies described above were the two procedures carried out in the same patients. The purpose of our report is to describe CT studies of the temporal lobe and SPET studies of perfusion of the cortex in the same group of patients. We wished to see whether there was any relation between atrophy of the medial temporal lobe and a perfusion deficit in the parietotemporal cortex and, if so, whether such a relation was associated with Alzheimer's disease defined clinically and, when possible, by histopathological criteria. Preliminary findings have been reported. $^{20}$

\section{Patients and methods}

Patients were recruited, after having been given a full explanation of the study protocol, from general practitioners and hospital based services, with some subjects entering themselves. Ethical approval for the study was obtained from both the Central Oxford Research and the Psychiatric Sector Research ethics committees. Informed consent was obtained from those without cognitive deficit, and for those with a significant deficit it was given by a close relative. Subjects were referred with varying degrees of mental deterioration and memory loss, many for detailed screening with a view to making a diagnosis. Diagnoses were made according to DSM-III-R criteria. Drug treatment remained unchanged during screening. 
Figure CT in the temporal lobe orientation of a patient who was not clinically demented (top) and a patient with a clinical diagnosis of senile dementia of the Alzheimer type ( $S D A T$ ) (bottom). The arrows indicate the thinnest part of the medial temporal lobe adjacent to the brain stem where a linear measurement was taken. The left of the brain is shown on the right, where there is also a scale in $\mathrm{cm}$. The non-demented patient was aged 80 at the time of the scan, while the patient with SDAT was 82 ; the respective right medial temporal lobe thicknesses were $17.5 \mathrm{~mm}$ and $10 \mathrm{~mm}$.

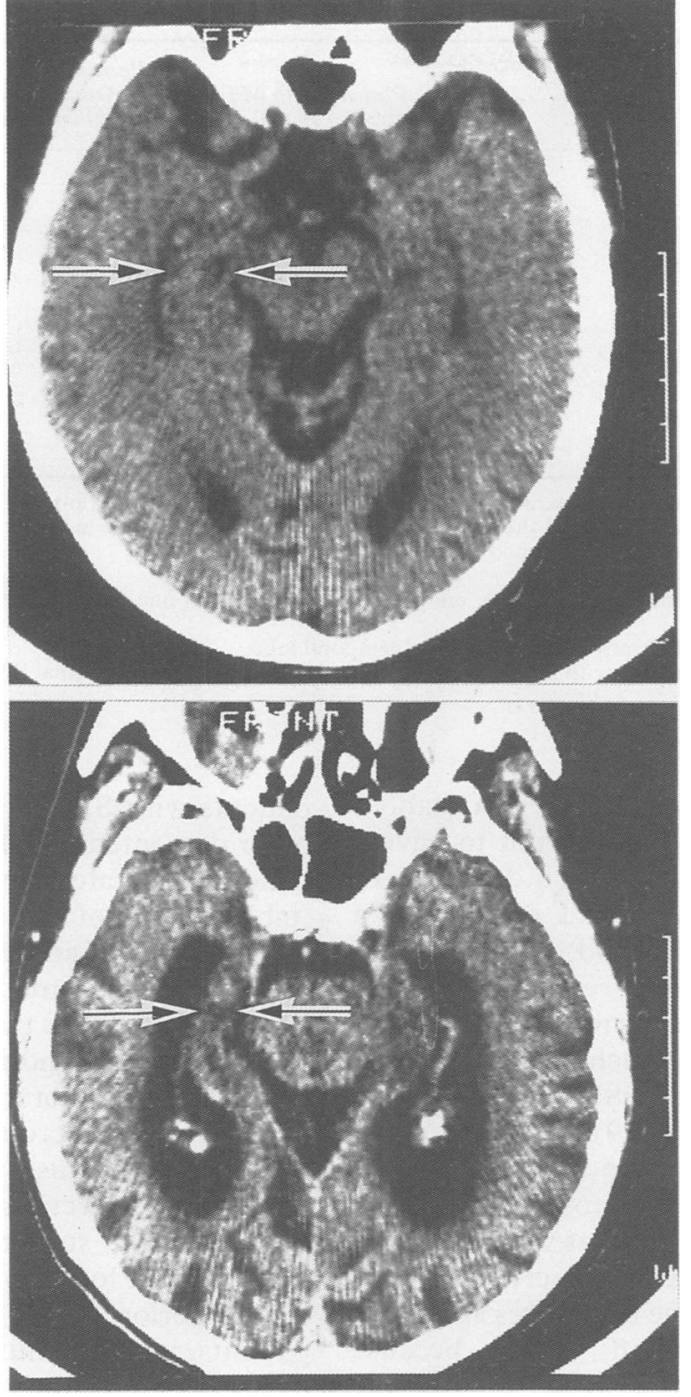

A detailed clinical and psychiatric history was taken from each subject, followed by a full physical examination of all systems, blood tests to exclude identifiable metabolic causes of memory loss and dementia, and neuropsychological screening with the Cambridge mental disorders of the elderly examination (CAMDEX). ${ }^{21}$ Screening specifically included CT and SPET of the brain.

CT scans were taken in the axial plane with $4 \mathrm{~mm}$ contiguous sections through the posterior fossa and $8 \mathrm{~mm}$ contiguous sections through the cerebrum parallel to the orbitomeatal line. After this procedure and starting with a lateral topogram sections were then taken passing through the front of the hard palate and top of the mastoids in $4 \mathrm{~mm}$ parallel sections through the temporal lobes; this scan plane is approximately 20 degrees caudad to the orbitomeatal line. Left and right medial temporal lobe width (combined hippocampal formation and parahippocampal gyrus) was then measured from the film, using calipers, at the narrowest point adjacent to the brain stem (figure 1). Measurements were made blind to the clinical diagnosis. All scans were evaluated by a neuroradiologist for evidence of any intracerebral pathology.
SPET studies were carried out with an intravenous injection of $500 \mathrm{MBq}$ hexamethylpropyleneamine oxime (HMPAO) labelled with technetium- $99 \mathrm{~m}$, which was given as a bolus and prepared according to the manufacturer's instructions (Ceretec, hexametazime, Amersham International). Sixty four SPET images were obtained over $360^{\circ}$, each having an acquisition time of $20 \mathrm{~s}$ and a resolution of $64 \times 64$ pixels. Axial and coronal slices were reconstructed by filtered back projection and were two pixels in width (13 mm). SPET scans were assessed blind to the clinical diagnosis on a scale of zero to three, in which 0 represented no perfusion deficit, 1 a minimal to mild deficit, 2 a moderate deficit, and 3 a severe perfusion deficit "breaching" the cortex. To localise and grade the deficits more accurately scans were assessed in both transverse and coronal planes. Grading was done alongside the CT scan so as to identify perfusion deficits in which intracerebral pathology was clearly discernible (such as a cerebral infarct) and accounted for the observed SPET perfusion anomaly. Any CT evidence of appreciable atrophy of the parietal lobe was also noted. SPET perfusion scores in the left, right, or bilateral parietotemporal regions with a grading of 2 or more were considered to indicate a clear perfusion deficit for the purpose of subsequent analysis.

Each subject was assessed with the CAMDEX. ${ }^{21}$ The cognitive section, CAMCOG, includes sections for testing memory, praxis, language, attention, concentration, orientation, abstract thinking, and calculation and has a maximum score of 107 . It also enables the derivation of the mini mental state examination, which is scored out of 30 . A score of less than 80 in the CAMCOG and of less than 24 in the MMSE was considered to indicate a significant cognitive deficit.

Neuropathological examination was performed on cerebral hemispheres that had been suspended in buffered neutral formalin for at least six weeks. Histological sections were prepared from the frontal, temporal, and parietal neocortex, hippocampus, cerebellum, midbrain, and pons. Sections were stained with haemotoxylin and eosin, congo red, luxol and fast blue cresyl violet and with methenamine silver $^{22}$ and Cross's modification of the Palmgren stain ${ }^{23}$ in order to demonstrate argyrophylic plaques and neurofibrillary tangles, respectively. Alzheimer's disease was diagnosed if the diagnostic criteria proposed. by Khachaturian were fulfilled, ${ }^{24}$ taking into account any clinical history of dementia.

\section{Results}

Clinical diagnosis

Fifty one of the 79 patients fulfilled the DSMIII-R criteria for SDAT and could be further categorised, as shown in the table, into those who were thought to have SDAT alone and those with other central nervous system disease as well. Ten patients had dementia not thought to be primarily SDAT, including five who initially presented with dementia but who had significant psychiatric symptoms consistent 
Table Results for patients in different diagnostic categories. Values are numbers of patients

\begin{tabular}{lcccc}
\hline Clinical diagnosis & $\begin{array}{l}\text { Total no } \\
\text { of patients }\end{array}$ & $\begin{array}{l}\text { Patients with MTL } \\
\text { atrophy on CT }\end{array}$ & $\begin{array}{l}\text { Patients with } \\
\text { parietotemporal } \\
\text { deficit on SPET }\end{array}$ & $\begin{array}{c}\text { Patients with both } \\
\text { CT and SPET changes }\end{array}$ \\
\hline SDAT: & & Clinically demented & & \\
$\quad$ Alone & 23 & 23 & 23 & 15 \\
With ischaemia & 19 & 18 & 15 & 6 \\
With other CNS disease & $9 \star$ & 8 & 3 & 3 \\
Multi-infarct & 3 & 3 & 0 & 0 \\
Progressive supranuclear palsy & 2 & 1 & 1 & 1 \\
Pseudodementia & 5 & 2 & & 2 \\
& & Not clinically demented & 2 & $1 \dagger$ \\
Lesion absent & 15 & 4 & $3 \ddagger$ & \\
Cerebrovascular accident & 3 & $1 \dagger$ & & \\
\hline
\end{tabular}

MTL = medical temporal lobe; patients were considered to have atrophy of the medial temporal lobe on CT if the thickness of one or both lobes was $\leqslant 11.5 \mathrm{~mm}$. A change in the SPET scan of the parietotemporal region on either side indicative of a moderate to severe reduced lobes was $\leqslant 11.5 \mathrm{~mm}$. A change in the SPET scan of the parietotempo

blood flow was defined by the scoring procedure

^Two with Parkinson's disease, two motoneurone disease, one Lewy body dementia, one frontal lobe dementia, one hypothyroid, two unknown.

$\dagger$ Cerebrovascular accident could account for atrophy of the medial temporal lobe.

$\ddagger$ wo with a cerebrovascular accident and one with previous surgery that could account for abnormal SPET scans.

with a diagnosis of pseudodementia (table). Eighteen patients were thought not to have a cognitive deficit consistent with a diagnosis of dementia, of whom two had CT evidence of a cerebrovascular accident and one had had neurosurgery in the parietal region.

Cognitive testing gave average scores for the demented group $(n=61)$ including those with pseuodementia of 51 (SD 28) (range 0-96) for CAMCOG and 15 (8) (0-29) for the MMSE; for the non-demented group $(n=18)$ the corresponding scores were 98 (6) (77-105) and $28 \cdot 5(1 \cdot 5)(25-30)$. Two patients were classified as demented by DSM-III-R criteria, even though they had cognitive test scores above the normally accepted cut off values, because estimates of their premorbid intelligence quotient (IQ) were extremely high; one of these patients had a clinical diagnosis of pseudodementia, the other a diagnosis of Alzheimer's disease and Parkinson's disease.

\section{Computed tomography}

The mean of the average thickness of left and right medial temporal lobes, measured at the thinnest point, in the 51 patients with SDAT was $8.5(2 \cdot 8) \mathrm{mm}(2 \cdot 75-15 \mathrm{~mm})$. The median value was significantly different $(p<0.0001$, Mann-Whitney) from that of the 18 nondemented patients, whose mean medial temporal lobe thickness was $14.7(3.0) \mathrm{mm}$ (range $10-18 \cdot 5)$. On this basis we defined a medial temporal lobe thickness of $11.5 \mathrm{~mm}$ or less as indicative of atrophy (figure). With this criterion 49 out of the 51 patients with SDAT had atrophy of the medial temporal lobe on one or both sides, as did six out of 10 of the other demented patients. However, only five out of 18 non-demented patients had medial temporal lobe atrophy; one of these five patients had evidence of cerebral infarction extending to the temporal lobe that could have accounted for the atrophy (table).

SPET

With the scale outlined above for scoring the SPET scans for the degree of perfusion deficit in the parietotemporal region, 44 out of the 51 patients with SDAT and four out of 10 of the other demented patients had a clear blood flow deficit (moderate to severe) on one or both sides (table). None of the demented group showed CT evidence of appreciable parietal lobe atrophy, focal ischaemia, or surgery that would have accounted for the changes seen on SPET. Five out of the 18 non-demented patients had a clear parietotemporal perfusion deficit, but the deficit could be accounted for in three of these by identifiable causes: two of the three had CT evidence of infarction in the parietal region and one had had surgery in the parietal lobe. Although the location of the perfusion deficit in the posterior cortex varied slightly between patients, it always included one or more of the following areas: the superior parietal lobe, the angular and supramarginal gyri, and the superior and middle temporal gyri.

\section{Comparison of CT and SPET changes in the same patient}

The occcurrence of reduced blood flow in parietotemporal regions as shown by SPET was invariably associated with atrophy of the medial temporal lobe as shown by CT: thus, all 50 patients who had clear parietotemporal perfusion deficits that could not be accounted for by infarction or surgery also displayed atrophy of one or both medial temporal lobes as shown by CT. On the other hand, atrophy of the medial temporal lobe was not invariably associated with reduced parietotemporal blood flow, although such an association was particularly striking in patients with a clinical diagnosis of SDAT (table). All 23 patients who were considered to have SDAT without other disease of the central nervous system displayed a combination of medial temporal lobe atrophy and clear parietotemporal perfusion deficit; CT scans from one of these patients and a nondemented patient are shown in the figure. Overall, only five of the 49 patients with SDAT who had atrophy of the medial temporal lobe did not show a marked perfusion deficit in the parietotemporal region (table); however, all five of these patients had a SPET perfusion score of 1 either on one or on both sides, which with our 
classification represented a mild perfusion deficit. Two patients classified as having SDAT as well as other disease of the central nervous system showed neither atrophy of the medial temporal lobe nor a clear perfusion deficit in the parietotemporal lobe, but both had unusual clinical features in addition to SDAT, which will be described in detail in a later report. Out of six demented patients considered not to have SDAT but who had atrophy of the medial temporal lobe, four also showed a parietotemporal perfusion deficit. The only one of these patients who died was found at necropsy to have Alzheimer's disease (see below).

In subjects in whom there was medial temporal lobe atrophy-that is, thickness of $11.5 \mathrm{~mm}$ or less on one or both sides-the left and right sides were compared to see whether there was any relation between asymmetrical medial temporal lobe atrophy and perfusion changes shown by SPET. Asymmetry of the atrophy was arbitrarily defined as a difference in thickness of $3 \mathrm{~mm}$ or more between the two sides. With this criterion, 12 of the 60 subjects with medial temporal lobe atrophy showed such an asymmetry (six on each side). In 10 (nine with SDAT) of these 12 patients SPET scans showed that the parietotemporal blood flow ipsilateral to the medial temporal lobe atrophy was lower than on the contralateral side. The remaining two subjects showed symmetrical perfusion deficits in the parietotemporal regions.

\section{Histopathology}

Ten of the 51 patients with a clinical diagnosis of SDAT died: nine fulfilled the histopathological criteria of Khachaturian for Alzheimer's disease $^{24}$ and the other was considered to have normal pressure hydrocephalus. All 10 of these patients showed the combination of medial temporal lobe atrophy and parietotemporal perfusion deficit. Two patients out of the 10 demented patients without SDAT died. One of these patients had a clinical diagnosis of multiinfarct dementia but had a histopathological diagnosis of Alzheimer's disease; this patient also showed medial temporal lobe atrophy and a parietotemporal perfusion deficit in life, as well as CT evidence of low density in the periventricular white matter. The other patient had a clinical diagnosis of progressive supranuclear palsy confirmed at necropsy, and did not show the combination of medial temporal lobe atrophy and parietotemporal perfusion deficit. Thus, so far, all 10 demented patients with histologically confirmed Alzheimer's disease showed a combination of the two changes.

\section{Discussion}

Our CT findings of atrophy of the medial temporal lobe are in agreement with several recent studies on demented patients who are presumed to have Alzheimer's disease, ${ }^{15} 16$ although we have used a relatively simple measurement that can be made directly from the film. Likewise, the results of our SPET studies agree with those of many published reports in which various tracers have been used to show reduced perfusion or uptake of tracer, particularly in the posterior parietotemporal region, of patients with a clinical diagnosis of Alzheimer's disease..$^{6-825-30}$

The striking feature of the results was the common occurrence of these two changes in the same patient. Both the CT and SPET changes often occurred in the same patient if the patient was clinically demented, particularly with dementia of Alzheimer's type. Eighty six percent $(44 / 51)$ of patients with dementia of Alzheimer's type had a combination of medial temporal lobe atrophy and reduced parietotemporal blood flow, while only four out of the 10 demented patients who did not have a clinical diagnosis of Alzheimer's disease showed this combination, one of whom was found to have Alzheimer's disease at necropsy (see below). Such a combination of changes was rare in patients who were not demented, occurring in only two out of 18 patients when we excluded the patient in whom the changes might be accounted for by focal ischaemic damage in the medial temporal lobe.

A combination of medial temporal lobe atrophy and reduced parietotemporal blood flow in the same patient may thus be indicative of Alzheimer's disease if due account is taken of clinical and CT evidence of ischaemic damage as a possible causative factor. Consistent with this suggestion is the finding that, of the 12 patients who died, the 10 with a histological diagnosis of Alzheimer's disease all displayed the combination of CT evidence of atrophy of the medial temporal lobe with SPET evidence of a parietotemporal perfusion deficit. It is noteworthy that the patient who died in the group classified clinically as having multiinfarct dementia in fact had a histological diagnosis of Alzheimer's disease and also displayed the combination of CT and SPET changes. We hope to follow all our patients through to necropsy to establish the histological diagnoses and, clearly, further combined CT and SPET studies should be carried out on patients with forms of dementia distinct from Alzheimer's disease.

A causal relation between the two changes may be indicated by the finding that in 10 out of 12 patients in whom the atrophy of the medial temporal lobe was more severe on one side than on the other, there was an identical asymmetry in the parietotemporal perfusion deficit. We suggest that the reduced parietotemporal blood flow might reflect pathological change in the projection neurones in the parahippocampal gyrus, which in monkeys ${ }^{31} 32$ innervate inferior parietal areas equivalent to the angular gyrus in man. ${ }^{33}$ Neurons that are pathologically affected in Alzheimer's disease are predominantly projection neurones that interconnect association and limbic areas of the cortex, ${ }^{134}$ pyramidal neurones of the hippocampal formation and parahippocampal gyrus being particularly affected. ${ }^{113}$ Medial temporal lobe atrophy in dementia might follow the loss of these projection neurones, whereas in other conditions with such atrophy (see introduction) it might be due to the loss of different neurones. Our finding suggests that, at least in the parietal lobe, one 
consequence of the damage or loss of projection neurones in the parahippocampal gyrus might be reduced metabolism and blood flow in the target area. As the disease progresses the loss of input from the hippocampal region might in turn lead to transneuronal pathological changes in the parietal lobe and consequent loss of tissue, which seems to be more severe in the later stages of Alzheimer's disease. ${ }^{935} 36$

We thank the patients, their carers, and the clinicians in the Oxford region who referred their patients for this study. Sue Stannard for coordinating many aspects of the study; and Drs M Esiri, J Marshall, and K McPherson for advice. BMcD held a Wellcome Research Training Fellowship during part of this work. The research was supported by a grant from the Bristol-Myers Squibb Company.

1 DeCarli C, Kaye JA, Horwitz B, et al. Critical analysis of the use of computer-assisted transverse axial tomography to study human brain in aging and dementia of the Alzheimer type. Neurology 1990;40:872-83.

2 Hoffman JM, Guze BH, Baxter LR, et al. <F-18> Fluorodeoxyglucose (FDG) and positron emission tomography (PET) in aging and dementia-a decade of studies. Eur Neurol 1989;29:16-24.

3 McGeer EG, Peppard RP, McGeer PL, et al. Fluorodeoxyglucose-18 positron emission tomography studies in presumed Alzheimer cases, including 13 serial scans. Can J Neurol Sci 1990;17:1-11.

4 Perani D, Di Piero V, Valler G, et al. Technetium-99mHMPAO-SPECT study of regional cerebral perfusion in HMPAO-SPECT study of regional cerebral perfusion in

5 Prohovnik I, Mayeux R, Sackeim HA, et al. Cerebral perfusion as a diagnostic marker of early Alzheimer's perfusion as a diagnostic marke
disease. Neurology 1988;38:931-7.

6 Burns A, Philpot MP, Costa DC, Ell PJ, Levy R. The investigation of Alzheimer's disease with single photon emission tomography. J Neurol Neurosurg Psychiatry 1989;52:248-53.

7 Hunter R, McLuskie R, Wyper D, et al. The pattern of function-related regional cerebral blood flow investigated by single photon emission tomography with Tc-99MHMPAO in patients with presenile Alzheimer's disease and Korsakoff's psychosis. Psychol Med 1989;19:847-55.

8 Montaldi D, Brooks DN, McColl JH, Patterson J, Barron E, McCulloch J. Measurements of regional cerebral blood flow and cognitive performance in Alzheimer's disease. flow and cognitive performance in Alzheim
$J$ Neurol Neurosurg Psychiatry 1990;53:33-8.

9 Brun A, Englund E. Regional pattern of degeneration in Alzheimer's disease: neuronal loss and histopathological Alzheimer's disease: neuronal loss and

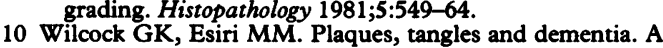
Wlcock GK, Esiri MM. Plaques, tangles and dem
quantitative study. $J$ Neurol Sci 1982;56:343-56.

11 Hyman BT, van Hoesen GW, Damasio AR, et al. Alzheimer's disease: Cell-specific pathology isolates the hippocampal formation. Science 1984;225:1168-79.

12 Ball MJ, Hachinski V, Fox A, Kirshen AJ, Fisman M, Blume W, et al. A new definition of Alzheimer's disease: a hippocampal dementia. Lancet 1985;i:14-6.

13 Esiri MM, Pearson RCA, Steele JE, Bowen DM, Powel TPS. A quantitative study of the neurofibrillary tangles and the choline acetyltransferase activity in the cerebral cortex and the amygdala in Alzheimer's disease. $J$ Neurol Neurosurg Psychiatry 1990;53:161-5.

14 LeMay $M$. CT changes in dementing diseases: a review. American Journal of Neuroradiology 1986;7:841-53.

15 Kido DK, Caine ED, LeMay M, et al. Temporal lobe atrophy in patients with Alzheimer's disease: a CT study. American Journal of Neuroradiology 1989;10:551-5.

16 de Leon MJ, George AE, Stylopoulos LA, Smith G, Miller DC. Early marker for Alzheimer's disease: the atrophic hippocampus. Lancet 1989;ii:672-3.

17 Esiri MM, Oppenheimer DR. Diagnostic neuropathology. Oxford: Blackwell Scientific Publications, 1989.

18 Roberts GW. Schizophrenia the cellular biology of a functional psychosis. Trends Neurosci 1990;13:207-11.

19 Press GA, Amaral DG, Squire LR. Hippocampal abnormalities in amnesic patients revealed by high-resonormalities in amnesic patients revealed by high-reso-
lution magnetic resonance imaging. Nature 1989;341: 54-7.

20 Jobst KA, Anslow PA, Barker S, et al. Diagnostic value of a combination of temporal lobe CT scans and SPET studies in dementia: definition of a subgroup of patients with structural and metabolic changes. Neurobiol Aging 1990; 11:262.

21 Roth M, Huppert FA, Tym E, et al. CAMDEX: the Cambridge examination for mental disorders of the elderly. Cambridge: Cambridge University Press, 1988.

22 Lamy C, Duyckaerts C, Delaere P, et al. Comparison of seven staining methods for senile plaques and patients. Neuropathol Appl Neurobiol 1989;15:563-78.

23 Cross RB. Demonstration of neurofibrillary tangles in paraffin section-a quick and simple method using paraffin section-a quick and simple method

24 Khachaturian ZS. Diagnosis of Alzheimer's disease. Arch Neurol 1985;42:1097-105.

25 Gemmell HG, Sharp PF, Evans NT, Besson JAO, Lyall D, Smith FW. Single photon emission tomography with ${ }^{123}$ Iisopropylamphetamine in Alzheimer's disease and multiinfarct dementia. Lancet 1984;ii: 1348.

26 Neary D, Snowden J, Shields R, Burjan AWI, Northen B, MacDermott N, et al. Single photon emission tomography using ${ }^{99 m}$ Tc-HM-PAO in the investigation of dementia. $J$ Neurol Neurosurg Psychiatry 1987;50:1101-9.

27 Perani D, Di Piero V, Valler G, et al. Technetium-99mHMPAO-SPECT study of regional cerebral perfusion in early Alzheimer's disease. $J$ Nucl Med 1988;29:1507-14.

28 Gemmell HG, Sharp PF, Smith FW, et al. Cerebral blood flow measured by SPECT as a diagnostic tool in the study of dementia. Psychiatry Res 1989;29:327-9.

29 Leys D, Steinling M, Petit H, et al. Single photon emission tomography with HMPAO Tc $c^{99 m}$ in Alzheimer's disease. tomography with HMPAO Tc $c^{9}$ in

30 Hellman RS, Tikofsky RS, Collier BD, et al. Alzheimer disease-quantitative analysis of I-123-iodoamphetamine SPECT brain imaging. Radiology 1989;172:183-8.

31 Mesulam MM, van Hoesen GW, Pandya DN, et al. Limbic and sensory connections of the inferior parietal lobule (area PG) in the rhesus monkey: a study with a new method for horseradish peroxidase histochemistry: Brain Res 1977;136:393-414.

32 Cavada C, Goldman-Rakic PS. Posterior parietal cortex in Rhesus monkey. 1. Parcellation of areas based on distinctive limbic and sensory corticocortical connections. J Comp Neurol 1989;287:393-421.

33 Eidelberg D, Galaburda AM. Inferior parietal lobule. Divergent architectonic asymmetries in the human brain. Divergent architectonic asymm
Arch Neurol 1984;41:843-52.

34 Pearson RCA, Esiri MM, Hiorns RW, et al. Anatomical correlates of the distribution of the pathological changes in correlates of the distribution of the pathological changes in the neocortex in Alzheim

35 Najerahim A, Bowen DM. Biochemical measurements in Alzheimer's disease reveal a necessity for improved neuroimaging techniques to study metabolism. Biochem $J$ 1988;251:305-8.

36 Bowen DM, Najlerahim A, Proctor AW, et al. Circumscribed changes of the cerebral cortex in neuropsychiatric disorders of later life. Proc Nat Acad Sci USA 1989;86: 9504-8. 